\title{
Helminthofaune des Teleostei de Madagascar. Révision du genre Pseudolamellodiscus Yamaguti, 1953
} (Monogenea)

\author{
par S. RAKOTOFIRINGA* et C. MAILLARD** \\ * Laboratoire de Biologie générale, E.E.S. Sciences. B.P. 906 Antananarivo, Madagascar. \\ ** et Laboratoire de Parasitologie comparée U.S.T.L. \\ pl. E.-Bataillon, F 34060 Montpellier Cedex.
}

\section{Résumé.}

Description de deux nouvelles espèces du genre Pseudolamellodiscus Yamaguti, 1953 (Monogenea, Diplectanidae): $P$. forsterii $\mathrm{n}$. sp. parasite des branchies de Sphyraena forsteri et $P$. jelloi parasite branchial de Shyraena jello. Etude des deux autres espèces de ce genre $P$. sphyraenae Yamaguti, 1953 et $P$. Nossibei Euzet et Razarihelisoa, 1959. Modification de la diagnose du genre.

\section{Summary.}

The helminthofauna of Teleostei from Madagascar. A review of the genus Pseudolamellodiscus Yamaguti, 1953 (Monogenea).

Two new species of the genus Pseudolamellodiscus Yamaguti, 1953 (Monogenea, Diplectanidae): $P$. forsterii $\mathrm{n}$. sp. and $P$. jelloi, branchial parasites of Sphyraena forsteri and Sphyraena jello respectively are described as well as the two other species of the genus, $P$. pshyraenae Yamaguti, 1953 and $P$. nossibei Euzet and Razarihelisoa, 1959. The diagnosis of the genus has been modified.

L'étude des Monogènes parasites des Téléostéens du genre Sphyraena péchés sur la Côte nord-ouest de Madagascar a permis de retrouver l'espèce type du genre Pseudolamellodiscus Yamaguti, 1953 ainsi que deux espèces nouvelles.

L'examen de ces helminthes et du matériel-type a conduit à réviser la diagnose du genre Pseudolamellodiscus.

Accepté le 26 mars 1979. 


\section{Pseudolamellodiscus forsterii n. sp.}

Hôte : Sphyraena forsteri Cuvier, 1829.

HABITAT: Branchies.

Localité : Nosy-be (Madagascar).

MATÉRIEl ÉtUdí́ : 10 individus étudiés in vivo. 30 individus colorés et montés in toto. 7 individus montés au Berlèse.

Type déposé au Museum national d'Histoire naturelle de Paris sous le $\mathrm{n}^{\circ} \mathrm{Tj} 36$. Mensurations (en $\mu \mathrm{m})$ :

Longueur du corps : 1150 (1 100-1 250).

Largeur du corps : 198 (180-240).

Hapteur : 190 (180-210).

Hamuli ventraux : $a=20 ; b=19,5(19-20) ; c=4,3 ; d=13 ; e=7$ (7-8).

Hamuli dorsaux : $a=16 ; b+d=24,5(24-25) ; e=9,5(9-10)$.

Barre ventrale : 187 (175-200).

Barres latérales : 68 (60-75).

Longueur du pharynx : 72,2 (70-75).

Largeur du pharynx: 50 (45-60).

Longueur du testicule : 275 (250-300).

Largeur du testicule : 95 (90-110).

Canal deferent : 190 (160-230).

Nombre de rangées du squamodisque : 50 à 56 .

MorPHOLOGIE (fig. 1).

Le corps allongé présente une région antérieure amincie où débouchent de chaque côté, de nombreuses glandes céphaliques. Les canaux de ces glandes ne sont pas réunis en faisceaux comme chez les autres Diplectanidae, mais forment deux bandes continues.

Le hapteur postérieur est aussi large que le corps.

Ce hapteur, procédé par deux squamodisques (un dorsal et un ventral) est armé de 2 paires de hamuli séparées par 3 barres transversales et de sept paires de crochetons latéraux disposés classiquement.

Chaque squamodisque se présente comme un organe semi-circulaire portant à la périphérie 50 rangées rayonnantes de 6 à 8 pièces sclérifiées placées bout à bout.

Le centre du squamodisque est occupé par des files d'épines prolongeant les rangées latérales.

Les hamuli dorsaux montrent une garde et un manche confondus en palette (fig. 3). 


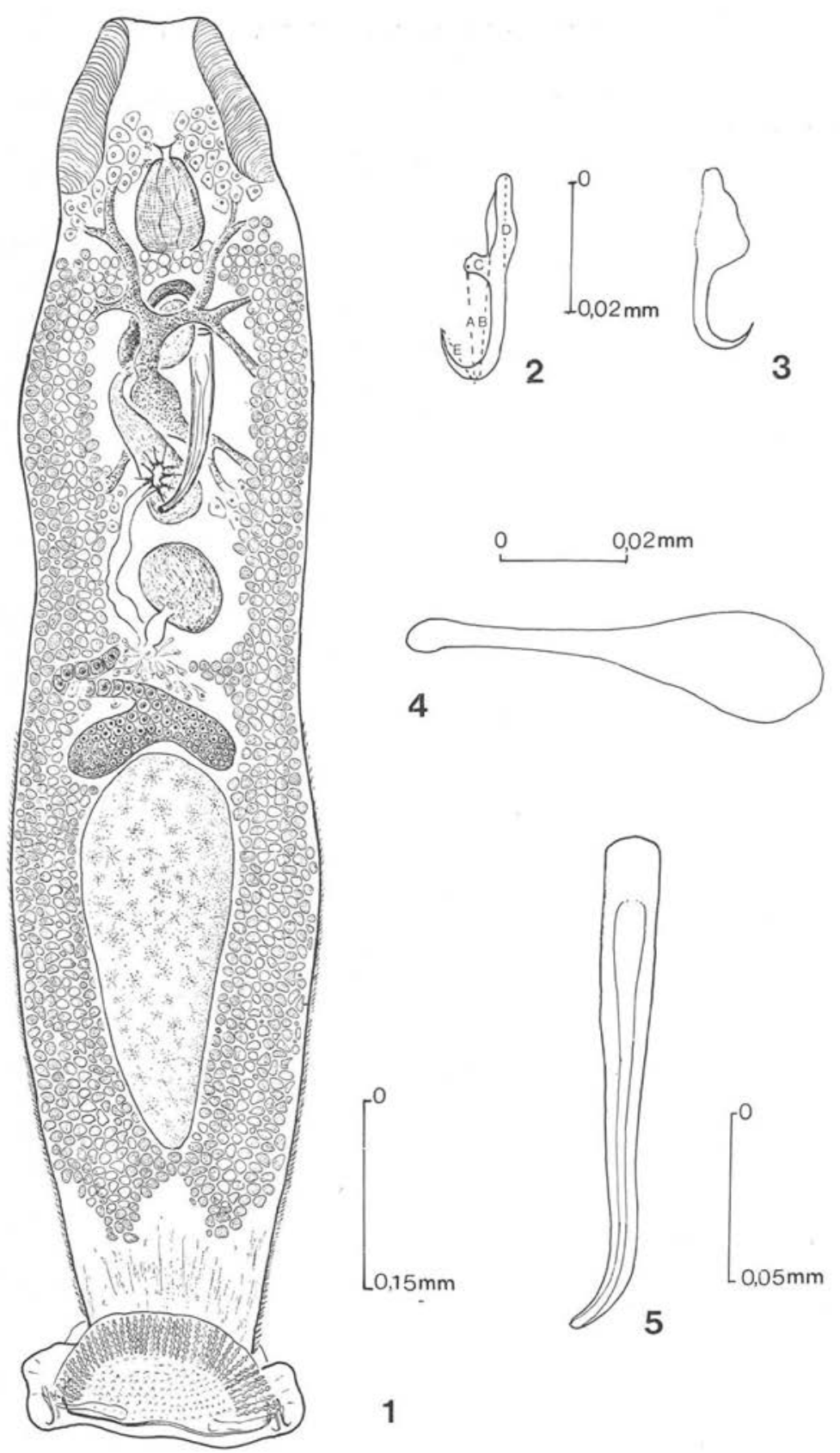

Fig. 1. Pseudolamellodiscus forsterii $\mathrm{n}$. sp. animal in toto vue ventrale.

Fig. 2. Pseudolamellodiscus forsterii n. sp.: Hamuli ventral.

Fig. 3. Pseudolamellodiscus forsterii n. sp.: Hamuli dorsal.

Fig. 4. Pseudolamellodiscus forsterii n. sp. : Barre transversale dorsale. Fig. 5. Pseudolamellodiscus forsterii n. sp.: Cirre. 
Les hamuli ventraux ont un manche long et massif ainsi qu'une garde plus réduite (fig. 2).

La barre transversale ventrale très longue, mince est plus ou moins enroulée à chaque extrémité.

Les barres transversales dorsales ont la forme d'une lame aplatie, élargie à l'extrémité axiale (fig. 4).

En avant du squamodisque, le corps est recouvert de minces écailles qui disparaissent au niveau équatorial. Il présente dans la région antérieure deux paires de taches oculaires dorsales, les postérieures étant les plus développées.

ANATOMIE (fig. 1).

\section{Appareil digestif.}

La bouche, ventrale, s'ouvre sur l'axe médian du corps au niveau des taches oculaires.

Le pharynx qui lui fait suite, piriforme, présente une lumière antérieure élargie où l'on distingue 3 paires de petites protubérances.

Après le pharynx, les deux branches digestives se séparent et longent les bords latéraux du corps. Elles se terminent séparément un peu en avant du hapteur.

Appareil génital femelle.

L'ovaire trilobé est situé dans la région équatoriale. Les deux lobes postérieurs coiffent le testicule et le lobe antérieur, plus allongé, entoure la branche droite de l'appareil digestif.

En avant de l'ovaire on distingue un volumineux réceptacle séminal subsphérique d'où se détache postérieurement un canal qui débouche dans le carrefour génital caché par les glandes de Mehlis.

De ce carrefour, l'utérus remonte vers l'avant dans l'espace intercaecal en longeant la branche digestive droite. Il se termine ventralement sur la ligne médiane par une fente longitudinale s'ouvrant sur le côté d'une dépression où aboutit aussi le cirre.

Les glandes vitellogènes, folliculaires, entourent les caecums digestifs depuis le pharynx jusqu’à leur extrémité distale.

Les vitelloductes transverses se détachent au niveau du carrefour génital où ils se rejoignent sur le plan médian.

Nous n'avons pu observer l'ouverture vaginale qui se situe peut-être au niveau de la dépression génitale.

Aucun œuf n'était présent dans l'utérus.

Appareil génital mâle.

Le testicule post-ovarien ovoïde et de grande taille occupe la moitié environ de l'espace intercaecal longitudinal.

Le canal déférent qui remonte dans cet espace intercaecal s'élargit en avant de l'ovaire pour donner une première vésicule séminale volumineuse. Après un retrécis- 
sement, une deuxième vésicule dorsale plus petite et à paroi musculaire vient déboucher à la base du cirre.

Le cirre, de forme conique très allongée, présente une extrémité distale amincie et courbée qui aboutit à une dépression médiane. Il est parcouru sur presque toute sa longueur par un étroit canal (fig. 5).

Le système prostatique est complexe. Les glandes prostatiques unicellulaires sont réparties en deux groupes dorsaux : un antérieur en avant et au-dessus du pharynx, l'autre postérieur au-dessus du cirre et la première vésicule séminale.

Les canaux collecteurs de ces deux groupes se réunissent pour former deux vésicules prostatiques ventrales qui débouchent à leur point de jonction dans une troisième vésicule dorsale se déversant à la base de l'organe copulateur.

Discussion,

Par la morphologie des pièces sclérifiées du hapteur et en particulier par la disposition de l'armature des squamodisques, ce parasite doit être placé dans le genre Pseudolamellodiscus Yamaguti, 1953.

Deux espèces $P$. sphyraenae Yamaguti, 1953 et $P$. Nossibei Euzet et Razarihelisoa, 1959 ont été décrites jusqu'à présent dans ce genre.

Cependant, l'anatomie du parasite de Sphyraena forsteri diffère de ces deux espèces par de nombreux caractères, en particulier par la forme très simple du cirre et l'absence des formations spéciales liées sans doute à la copulation que l'on note chez les espèces précédemment citées.

Nous la considérons comme une espèce nouvelle et nous proposons de la nommer Pseudolamellodiscus forsteri $\mathrm{n}$. sp.

\section{Pseudolamellodiscus jelloi n. sp.}

HôTE : Sphyraena jello Cuvier, 1829.

HaBitat : Branchies.

Localité : Nosy-be (Madagascar).

MatérIel Étudié : 30 individus étudiés in vivo. 18 colorés et montés in toto. 6 montés au Berlèse.

Mensurations (en $\mu$.).

Longueur du corps: 1050 (900-1 150).

Largeur du corps : 150 (150-160).

Hapteur: 275 (220-300).

Hamuli ventraux : $a=23(22-24) ; b=24(24-25) ; c=3 ; d=19$ (18-19); $e=6$.

Hamuli dorsaux : $a=18(18-19) ; b+d=31(30-32) ; e=6$. 
Barre ventrale : 235 (210-275).

Barres dorsales : 74 (65-85).

Longueur du pharynx: 64 (50-75).

Largeur du pharynx: 43 (30-50).

Longueur du testicule : 80 (75-85).

Largeur du testicule : 50 (45-50).

Longueur du canal déférent : 140 (135-150).

Nombre de rangées de squamodisques : 60 à 70 .

MoRPHOLOGIE (fig. 6).

Ce parasite, de petite taille, a un corps allongé aplati dorso-ventralement. L'extrémité antérieure moins large, porte de chaque côté de nombreuses glandes céphaliques disposées en 2 bandes latérales et dorsalement deux paires de taches oculaires.

Nous n'avons pas observé d'écailles ni d'épines tégumentaires.

La face ventrale du corps présente une forte dépression médiane au niveau des ouvertures génitales et l'on peut noter sur le côté droit, une petite cavité aveugle à parois épaisses et sclérifiées dont la fonction est énigmatique.

Le hapteur postérieur est étiré transversalement et déborde largement de chaque côté du corps. Il est muni de deux squamodisques (un dorsal et un ventral) de deux paires d'hamuli séparées par trois barres transversales et de sept paires de crochetons marginaux.

Chaque squamodisque paraît formé de deux demi-disques, contigus sur le plan médian et dont les diamètres sont alignés. Les rangées de pièces sclérifiées au nombre de trente-cinq par demi squamodisque sont concentriques par rapport à un point situé à la jonction des deux demi périmètres, la concavité de ces rangées est donc tournée vers le plan médio-sagittal du corps.

Le nombre des pièces sclérifiées varie de 4 à 14 en allant de ce plan vers la périphérie.

Les hamuli et les barres transversales ont sensiblement la même forme que ceux de $P$. forsteri (fig. $7,8,9$ ).

Anatomie (fig. 6):

\section{Appareil digestif.}

Il débute par une bouche ventrale, médiane, placée en-dessous des taches oculaires, un pharynx piriforme et deux caecums digestifs latéraux qui se terminent séparément en avant du hapteur.

Appareil génital femelle.

L'ovaire, situé dans le tiers postérieur du corps, entoure la branche digestive droite avec son extrémité antérieure. 


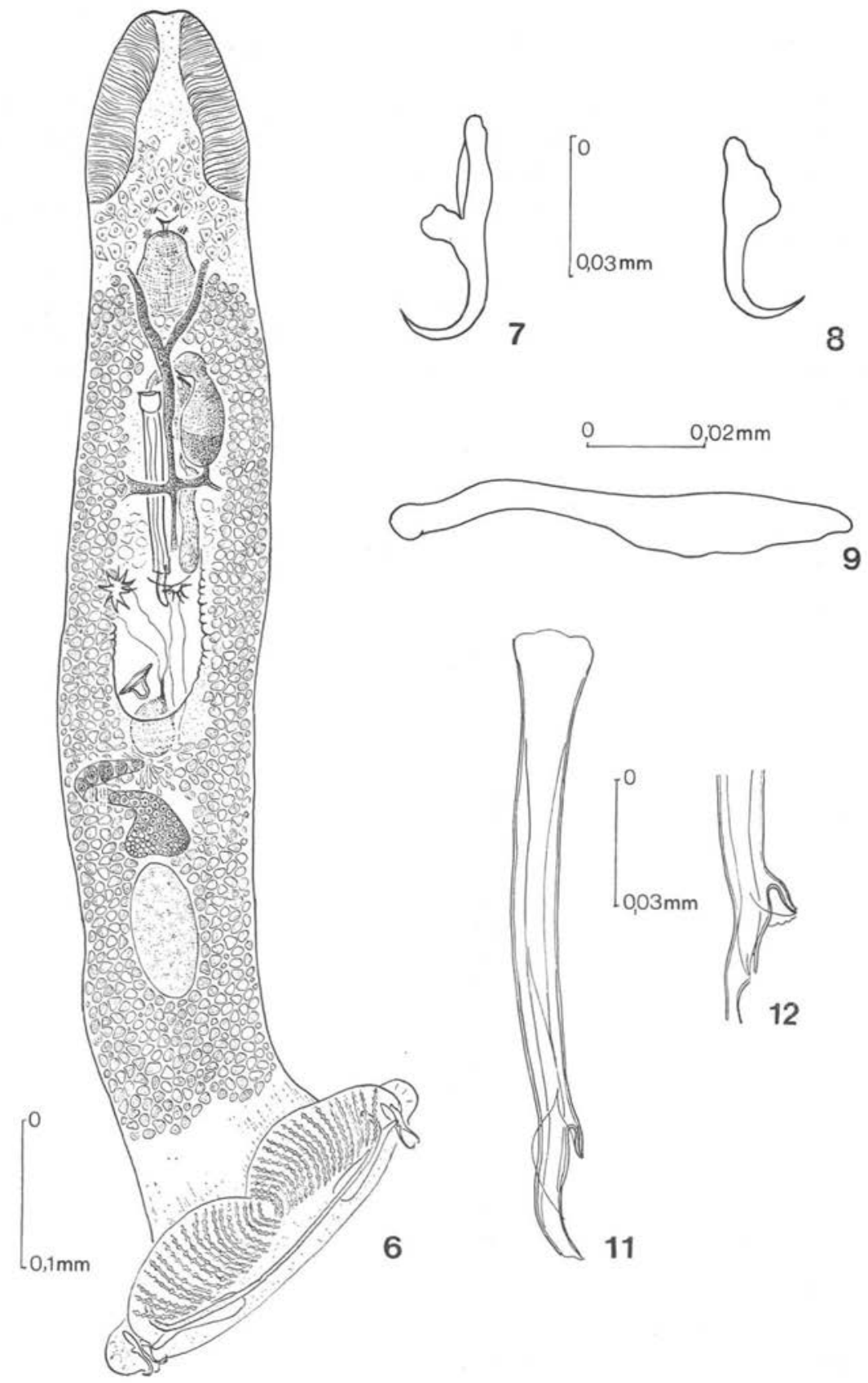

Fig. 6. Pseudolamellodiscus jelloi $\mathrm{n} . \mathrm{sp}$. : Animal in toto en vue ventrale.

Fig. 7. Pseudolamellodiscus jelloi n. sp.: Hamuli ventral.

Fig. 8. Pseudolamellodiscus jelloi n. sp.: Hamuli dorsal.

Fig. 9. Pseudolamellodiscus jelloi n. sp.: Barre transversale dorsale.

Fig. 11. Pseudolamellodiscus jelloi n. sp.: Cirre.

Fig. 12. Pseudolamellodiscus jelloi n. sp.: Extrémité distale du cirre de certains individus. 
Le réceptacle séminal sphérique est placé en avant de l'ovaire sur la ligne médiane. Il reçoit à sa partie antérieure le vagin qui débute ventralement au niveau équatorial à égale distance entre le plan médian et le côté droit du corps.

Tout d'abord, très large et à parois plissées, il se rétrécit brusquement pour donner un fin canal à paroi sclérifiée qui débouche dans le réceptacle séminal.

Chez certains individus, nous avons noté, dans la chambre antérieure du vagin, la présence d'un spermatophore volumineux (fig. 10). Il se présente comme un sac ovoïde possédant à un pôle un long filament dont la majeure partie est repliée à l'intérieur d'une chambre représentant environ le $1 / 3$ du spermatophore, le reste étant occupé par les spermatozoïdes.

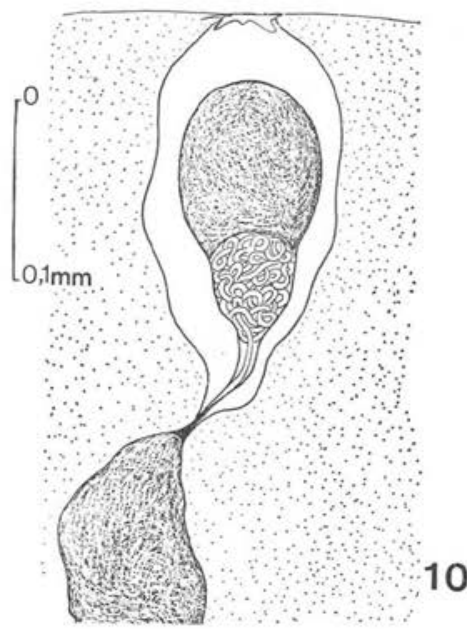

Fig. 10. Pseudolamellodiscus jelloi n. sp. Spermatophore dans le vagin.

Le fonctionnement de cette formation, et en particulier celui du filament pelotonné dans la chambre polaire demeure énigmatique.

L'utérus remonte depuis le carrefour génital jusqu'à son ouverture placée ventralement au milieu du corps.

Les glandes vitellogènes folliculaires entourent les branches digestives depuis leur naissance jusqu'à leur extrémité postérieure.

L'œuf de ce Monogène est inconnu.

Appareil génital mâle.

Le testicule ovoïde est situé dans l'espace intercaecal, en arrière de l'ovaire.

Un peu en avant des ouvertures génitales, le canal déférent se renfle puis donne une vésicule séminale sacculaire qui se jette à la base du penis.

Celui-ci a la forme d'un tube évasé à sa partie proximale et aminci à son extrémité distale. Cette extrémité est chez certains individus recourbée en crochet (fig. 11) et chez d'autres sub-rectiligne (fig. 12). 
Seule une étude statistique pourrait déterminer si ce détail morphologique correspond à un caractère spécifique ou si cela traduit des variations intraspécifiques.

Un peu avant l'extrémité distale du cirre se distingue une collerette sclérifiée soutenue par une expansion de la paroi du tube.

Le cirre est parcouru dans sa totalité par un canalicule à paroi mince.

Le système prostatique comprend deux groupes de glandes dorsales, le premier un peu en avant du pharynx, le second au-dessus de la région occupée par le cirre

Les canaux de ces glandes s'unissent pour former quatre canaux collecteurs ( 1 antérieur, 1 postérieur et 2 latéraux) qui se rejoignent avant de déboucher à la base d'une vésicule prostatique bipartite.

Les sécrétions contenues dans cette vésicule sont de 2 types et se déversent à la base du cirre.

Discussion.

Cette espèce récoltée sur Sphyraena jello est très voisine de Pseudolamellodiscus nossibei décrit par Euzet et Razarihelisoa en 1959 chez Sphyrna commersoni Cuvier, 1829 , mais elle en diffère par un certain nombre de caractères.

$P$. nossibei est plus trapue avec un hapteur élargi dont les squamodisques portent 100 à 120 rangées de pièces sclérifiées alors que nous n'en avons compté que 60 à 70 chez l'espèce de Sphyraena jello.

Nous pensons que cette espèce est nouvelle et nous proposons de la nommer :

$$
\text { Pseudolamellodiscus jelloi n. sp. }
$$

En 1959, Euzet et Razarihelisoa dans leur description de P. nossibei signalent l'ovaire comme entourant la branche digestive gauche. Nous avons toutefois pu vérifier sur leurs préparations que l'ovaire entoure bien la branche digestive droite comme cela semble être la règle pour toutes les espèces du genre Pseudolamellodiscus. De plus, l'ouverture vaginale et l'ouverture génitale, non précisées par ces auteurs, occupent la même position que chez Pseudolamellodiscus jelloi n. sp.

\section{Pseudolamellodiscus sphyraenae (Yamaguti, 1953)}

Hôtes: Sphyraena sp. Sphyraena barracuda (Walbaum, 1972).

HaBitat : Branchies.

Localités: Macassar - Nossy-be (Madagascar).

MATÉRIEL ÉTUdí́ : 15 individus étudiés in vivo. 20 individus colorés et montés in toto. 4 individus montés au Berlèse.

Mensurations en $\mu \mathrm{m}$ :

Longueur du corps: 1100 (800-1 600). 
Largeur du corps : 165 (130-200).

Hapteur: 225 (215-250).

Barre ventrale : 250 (215-280).

Barres dorsales : 65 (55-75).

Hamuli ventraux : $a=19(19-21) ; b=22(20-25) ; c=4(3-5) ; d=16$ $(17-18) ; e=5$ (4-6).

Hamuli dorsaux : $a=18$ (17-19); $b+b=31$ (30-32); $e=6(6-7)$.

Pharynx longueur: 73 (65-80); largeur: 46 (30-50).

Testicule longueur: 120 (110-130); largeur: 57 (50-60).

Canal déférent : 165 (150-180).

Pièces sclérifiées: pièce gauche : 35 ; pièce droite : 20 .

Nombre de rangées de pièces sclérifiées du squamodisque : 46 à 54 .

Sur les cinq spécimens de Sphyraena barracuda examinés, nous avons récolté un nombre élevé de Monogènes appartenant à l'espèce Pseudolamellodiscus sphyraena Yamaguti, 1953.

L'étude d'une quarantaine d'individus nous a permis de comparer ce matériel avec la description très détaillée donnée par Yamaguti. Celle-ci correspond à nos observations en particulier en ce qui concerne l'appareil prostatique complexe, les formations sclérifiées carastéristiques, la forme du cirre, etc. (fig. 13).

Cependant, quelques détails anatomiques demandent à être complétés.

Ainsi, il est certain que l'ovaire entoure la branche digestive droite, disposition constante chez les animaux de ce genre.

Yamaguti dénombre 27 rangées d'écailles sur chaque squamodisque et en représente 51 dans son iconographie. Chez nos spécimens nous en avons compté 46 à 54 .

Dans la description originale, Yamaguti s'interroge sur la fonction des organes «Sui generis » qu'il observe chez cet animal.

Nous pensons que ces formations jouent un rôle lors de l'accouplement.

On peut imaginer, en effet, que celui-ci se déroule alors que les animaux sont placés face à face. Dans cette position, la pièce en forme de massue située sur le côté droit se trouve opposée à la cavité munie de 5 à 6 épines, placée sur le côté gauche du partenaire et dans laquelle elle s'insère.

Nous avons pu observer que ces épines pouvaient s'ouvrir et se refermer à la manière d'un parapluie permettant une préhension.

De plus, il est possible que la barre sclérifiée présente le long de l'ouverture vaginale s'insinue entre les deux branches du pénis augmentant ainsi la solidité de la fixation au moment de l'accouplement. 


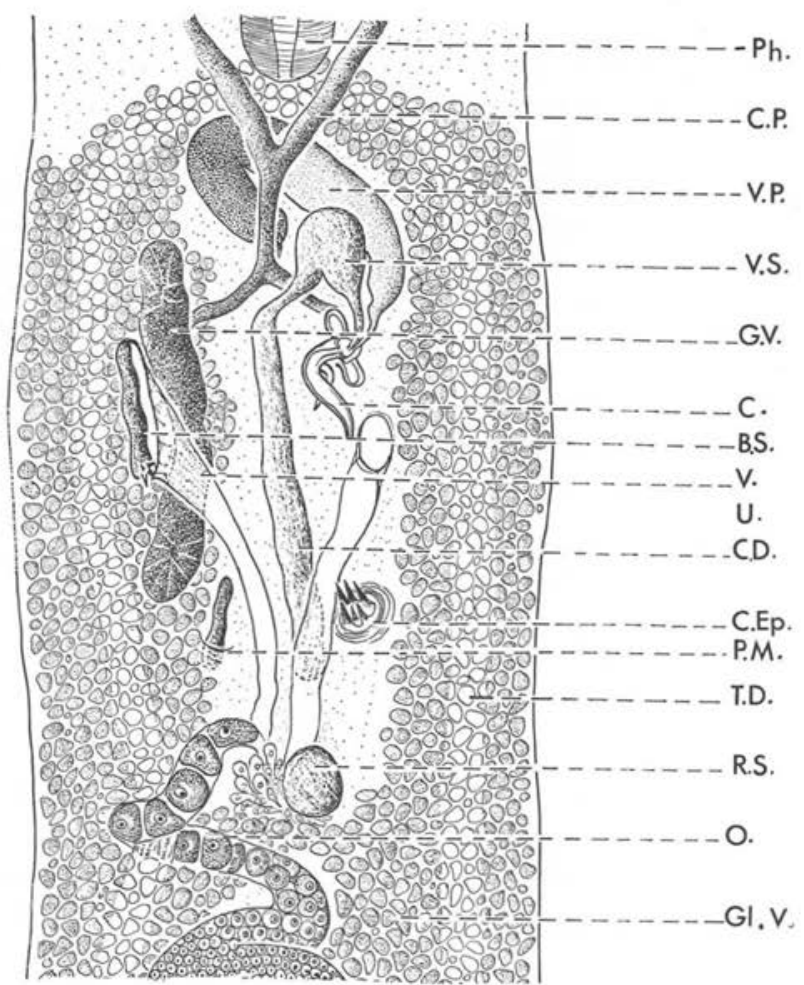

Fig. 13. Pseudolamellodiscus sphyraenae Yamaguti, 1953: Détail : vue ventrale.

B.S.: Barre sclérifiée.

C. : $\quad$ Cirre.

C.D.: Canal déférent.

C. Ep.: Cavité épineuse.

C.P.: Canal prostatique.

G.V.: Glande vaginale.

Gl. V.: Glandes vitellogènes.

O.: Ovaire.
Ph.: $\quad$ Pharynx.

P.M. : $\quad$ Pièce en massue.

R.S.: Réceptable séminal.

T.D.: Tube digestif.

U.: $\quad$ Utérus.

V.: Vagin.

V.P.: Vésicule prostatique.

V.S.: Vésicule séminale.

\section{Conclusion}

L'étude de Pseudolamellodiscus forsterii n. sp., $P$. jelloi n. sp., $P$. sphyraenae Yamaguti, 1953 récoltés sur les Sphyraena des côtes Malgaches ainsi que l'examen du matériel type de $P$. nossibei Euzet et Razarihelisoa, 1959 nous permettent de modifier et de préciser la diagnose du genre.

\section{Genre Pseudolamellodiscus Yamaguti, 1953.}

Corps allongé de petite taille. Hapteur muni de sept paires de crochetons, de deux paires d'hamuli latéraux séparés par deux barres transversales dorsales et une barre 
transversale ventrale très mince repliée en crosse aux deux extrémités. Deux squamodisques (un dorsal et un ventral) formés de plusieurs rangées rayonnantes de petites pièces sclérifiées. 2 paires de taches oculaires dorsales. Caecums intestinaux terminés séparément. Canal déférent médian. Vésicule séminale et vésicule prostatique présentes. Glandes prostatiques réparties en deux champs dorsaux. Organe copulateur sclérifié. Ovaire prétesticulaire entourant la branche droite de l'appareil digestif. Vagin et réceptacle séminal présents. Glandes vitellogènes folliculaires entourant les caecums digestifs depuis leur origine jusqu'à leur extrémité postérieure.

Parasites branchiaux de Téléostéens Sphyraenidae.

Le genre Pseudolamellodiscus semble inféodé aux Téléostéens du genre Sphyraena.

De plus, chaque espèce de Sphyraena paraît être l'hôte d'une espèce particulière de Pseudolamellodiscus.

Nous avons en effet récolté :

$P$. forsterii uniquement sur Sphyraena forsteri.

$P$. jelloi que sur Sphyraena jello.

$P$. sphyraena que sur Sphyraena barracuda et l'espèce $P$. nosibei n'a été observée que sur $\tilde{S}$. commersoni.

Compte tenu de cette spécificité parasitaire, l'espèce d'hôte-type du genre Pseudolamellodiscus non précisé par Yamaguti pourrait être $S$. barracuda.

Il en résulte que la mise en synonymie entre $S$. barracuda et $S$. commersoni proposée par Abe, 1974 devrait être reconsidérée.

\section{Bibliographie}

Abe T. (1974): Eastern Indian Ocean and Western Central Pacific. Fao identification sheets. W et P. J.P. Fischer, édit., Whithead.

Euzet L., Razarihelisoa M. (1959) : Sur quelques Monogènes de Sphyraena commersonii (Teleostei Sphyraenidae). Bull. Soc. Zool. Fr., 84, 77-85.

Yamaguti S. (1953) : Parasitic worms mainly from Celebes. Part. 2. Monogenetic Trematodes of fishes. Acta Med. Okayama, 8, 204-256. 